\title{
FACTORES QUE INCIDEN EN EL PROCESO DE ENSEÑANZA DE LA LECTURA Y LA ESCRITURA
}

Verónica Ramayón y Arianna Vignole

\section{INTRODUCCIÓN}

De acuerdo con las nuevas corrientes y con los estudios que se realizan permanentemente en el área de la lectura y la escritura, nos resultó interesante conocer cómo emprenden la enseñanza de ambas, los docentes de primeros años de primaria. Particularmente nos pareció significativo poder identificar algunos factores que pueden estar incidiendo en la enseñanza de la lengua escrita.

En base a esto nos preguntamos: ¿qué factores toma en cuenta el docente para la enseñanza de la lectura y la escritura?

\section{MARCO TEÓRICO}

En primer lugar consideramos importante definir el concepto de enseñanza; etimológicamente enseñar significa mostrar algo a alguien. Se considera la forma normal de la instrucción cultural sistemática, pero no a toda instrucción le llamamos enseñanza. Según Dubois(1991), cuando se habla de que la escuela debe enseñar a leer y a escribir, el sentido que se le da a la palabra "enseñar" es el de un " hacer que alguien aprenda algo", lo cual entraña un elemento de imposición frente al sujeto que aprende. Tradicionalmente la enseñanza de la lectura y la escritura ha tomado la forma de algo impuesto al niño desde fuera por decisión de los adultos que actuamos como docentes. Esta actitud llamada adultocentrismo por Ferreiro (1987) nos conduce no solo a fijar el qué, cómo y cuándo, sino a decidir qué es lo fácil o difícil para el niño, con el grave riesgo de que nuestra perspectiva pueda diferir de la del niño.

El sentido de enseñar puede sin embargo ser entendido de otra manera, como un "mostrar algo", en cuyo caso desaparece la característica de cosa impuesta desde afuera. La idea de enseñar la lectura en esta última forma es perfectamente aceptable desde el punto de vista de las nuevas concepciones de la misma, porque implicaría mostrar al niño la manera en que los adultos utilizan la lectura.

La escuela, pues, enseña a leer y escribir, siempre que se acepte que enseñar es "mostrar" antes que imponer y que lo que el niño necesita, entre otras cosas, es la guía adecuada y oportuna del maestro. Según Ferreiro y Palacios (1990) es necesario examinar el papel de la escuela como uno entre otros muchos elementos que contribuyen, dentro del medio ambiente de cada individuo, al aprendizaje de la lectura.

Para Smith (1990) todos los métodos de enseñanza de la lectura permiten alcanzar algún resultado, con algunos niños, algunas veces. Todas las investigaciones de enseñanza de la lectura muestran que ninguno de los métodos desarrollados alcanza a todos los niños. La responsabilidad del maestro no radica en enseñar a los niños a leer, sino en posibilitarles que aprendan a leer; facilitar el aprendizaje de la lectura, equivale a hacer de la lectura una experiencia significativa, placentera, útil y frecuente para los niños(Ferrá, 1993). 
En los últimos años, en el campo teórico de la lectura, se ha venido desarrollando el concepto de transacción para referirse al proceso por el cual el lector construye un texto paralelo y estrechamente relacionado con el texto editado, pero no idéntico al que el autor tenía en su mente antes de expresarlo por escrito. Entre el lector y el texto se da un proceso de transacción a través del cual ambos se tranforman.

Con referencia a la escritura, hoy sabemos que se la está presentado como un proceso que involucra aspectos lingüísticos y cognitivos. Escribir es construir sentido, representarlo gráficamente con una intención determinada (Salvo, 1994). Los aspectos psicomotrices, que fueron dominantes en épocas pasadas, vienen siendo reemplazados por aspectos que se vinculan más con la semántica y la pragmática del disurso.

La bibliografía tradicional reconocía una serie de "pre-requisitos" para el aprendizaje de la lengua escrita: fisiológicos, psicológicos, motrices, etc. Estos pre-requisitos, con frecuencia se transformaban en factores a tener en cuenta en la intervención del docente.

\section{OBJETIVOS}

\section{Objetivo general}

Contribuir a conocer los factores que los docentes tienen en cuenta en la enseñanza de la lengua escrita en primer año de educación primaria.

\section{Objetivo específico.}

Hacer de la investigación un instrumento de reflexión y cambio en manos del docente.

\section{CAMPO DE ACCIÓN}

La investigación se desarrolló en una institución privada, trilingüe, ubicada en una zona residencial de la ciudad de Montevideo. Los sujetos de la investigación fueron los docentes y los alumnos de primeros años del colegio.

El abordaje fue básicamente cualitativo. Según Taylor y Bogdan (1987) esta metodología se refiere a la investigación que produce datos descriptivos: las propias palabras de las personas, habladas o escritas y la conducta observable se convierten en elementos relevantes. La concepción de la metodología cualitativa se inscribe dentro de la perspectiva fenomenológica. La tarea del fenomenólogo es aprehender los fenómenos sociales desde la propia perspectiva del autor. Para él lo que la gente hace o dice es producto del modo en que define el mundo.

\section{DESCRIPCIÓN DE LOS INSTRUMENTOS METODOLÓGICOS}

Las técnicas que se utilizaron para la colecta de datos fueron las entrevistas y las observaciones. Se entrevistaron a las maestras de los primeros años y se llevaron a cabo observaciones en las distintas clases de primer grado. La entrevista no estructurada, que fue la seleccionada, es considerada una técnica muy productiva ya que no se ajusta estrictamente a la fórmula pregunta-respuesta, sino que se detiene en comentarios, descripciones y confirmaciones. Esto permitió esclarecer experiencias, ideas y visiones de los sujetos entrevistados. Las observaciones fueron realizadas con un propósito definido, dirigidas sistemática y cuidadosamente y registradas en su totalidad. Tanto la validez como la fiabilidad de la observación mejoran cuando éstas se hacen a intervalos frecuentes por el mismo observador o cuando distintos observadores recogen sus observaciones con independencia (Taylor y Bogdan, 1987).

42 - Universidad ORT Uruguay 
En nuestro caso tuvimos reiterados encuentros con los sujetos de la investigación a efectos de observar el desarrollo de las clases. Se establecieron algunas pautas de observación, en los demás casos se optó por un registro directo de campo.

Tanto las entrevistas como las observaciones fueron probadas, con la intención de verificar su pertinencia para la recolección de la información.

La colecta de datos se desarrolló según el cronograma establecido; en términos generales hubo buena disposición para colaborar con el trabajo. El hecho de haber realizado estudios pilotos con los instrumentos, nos permitió una más apropiada recolección de la información.

\section{ANÁLISIS DE LOS DATOS}

Al realizar el análisis de los datos fue posible elaborar las siguientes consideraciones:

. Las concepciones de lectura y escritura manejadas por los docentes se inscriben dentro de paradigmas algo distantes a los que nosotras presentamos en el marco teórico. En los primeros se percibe a la lectura como un acto mecánico que debe ser enseñado paso a paso; para nosotras, la lectura es interrogación de texto (Jolibert, 1993) y la tarea del docente es la de facilitador de instancias de interacción de los niños con los textos, además de informante competente sobre los procesos implicados en el acto de lectura.

. De igual manera, con referencia a la escritura, los datos recolectados nos informan de concepciones muy próximas a lo psicomotriz, no poniendo mucho énfasis en aspectos semánticos y pragmáticos.

. Factores fisiológicos, ambientales, emocionales e intelectuales de alguna manera emergen en los datos como aspectos relevantes en el momento de diseñar el proyecto de enseñanza de la lengua escrita.

. La presencia de estos factores, y su incorporación a la propuesta de enseñanza, explica de algún modo las concepciones de lectura y escritura dominantes en el colectivo docente.

. La mayoría de las prácticas en lengua escrita, desarrolladas por los niños e impulsadas por las concepciones de enseñanza de los docentes, se pueden vincular con posturas mecanicistas de la lectura y la escritura.

\section{REFLEXIONES FINALES}

Estamos en un proceso de cambio. La formación y la actualización de docentes requiere de tiempos. Tiempos para estudiar, tiempos para intercambiar, tiempos para dudar. En nuestro contexto de formación ha pesado mucho la postura mecanicista, segmentarista del saber; nos resulta muy difícil modificar nuestras prácticas de aula. No obstante, las nuevas investigaciones en el área, como el sentido común que emana de nuestra práctica diaria, nos informan que la enseñanza de la lectura y la escritura, entre otras cosas, debe atender al ritmo de cada niño, no siendo el docente el que decide el momento en que éste está maduro. 
En fin, se trata de un docente que sabe esperar a los niños, pero su espera no debe ser pasiva. Se puede esperar porque se comprende cuál es el proceso del pensamiento en el sujeto que aprende, pero teniendo en cuenta que las transformaciones de ese proceso no se suceden mágicamente, sino que son el producto de las interacciones con el medio social y material en el cual se incluye el sujeto.

Consideramos de suma importancia la actualización de los docentes, el permanente estudio y el constante perfeccionamiento, ya que el cambio de actividades no determina por sí solo el cambio de modalidad didáctica; la actividad no define la didáctica.

\section{Bibliografía}

DUBOIS, M.E. 1991. El proceso de lectura. Bs. As., Aique.

FERRÁ,C. 1993. Aprendizaje de la lectoescritura; Montevideo, Amauta.

FERREIRO, E. 1987. Los sistemas de escritura en el desarrollo del niño. México D.F., Siglo XXI.

FERREIRO, E. y Palacios, M. 1990. Nuevas perspectivas sobre los procesos de la lectura y la escritura. México D.F., Siglo XXI.

JOLIBERT, J. 1992. Formar niños productores de textos. Santiago de Chile, Hachette.

JOLIBERT, J. 1993. Formar niños lectores de textos. Santiago de Chile, Dolmen.

POSTIC, M. y DE KETELE, J. 1988. Observar las situaciones educativas. Madrid, Narcea.

SALVO, H. 1994. Contexto de la lecto-escritura: entre el alfabeto y el texto. Montevideo, Amauta.

SMITH, F. 1990. Para darle sentido a la lectura. Barcelona, Visor.

TAYLOR, S. y BOGDAN, R. 1987. Introducción a los métodos cualitativos de investigación. Bs. As., Paidós. 